\title{
Experimental Study of Wireless Anti-removed Identification Positioning Card (WAIPCS)
}

\author{
He Shengping \\ P.O.Box 150-11 \\ Shannxi Baoji, China \\ heshengping@163.com
}

Gao Mengji

School of Computer Science and Technology, Xi'an

University of Science and Technology

Shannxi Xi' an , China

moonlight_magician@126.com

\author{
Zhang Xin \\ P.O.Box 150-11 \\ Shannxi Baoji , China \\ npuzx@163.com
}

\begin{abstract}
It 's required that specific monitoring system should be capable to positioning the controlled personnel within monitoring area,meantime the staff location and status information could be sent to the monitoring center. The positioning signal card not only be used to transmit the controlled personnel status monitoring information, but also be easy to carry and anti- removed. This paper describes experimental works and relevant trials research of a kind of wearable wireless anti-removed identification positioning card system (WAIPCS). Experimental includes test of heart rate reading function, test basic function of prototype of WAIPCS, experiment for positioning function. Test of heart rate reading function showed that the actual human heart rate is kept with and meet the design requirements. Test basic function of prototype of WAIPCS showed that normal unauthorized event alarm function has been realized in the device, and the system could read the Id information reliably within the distance $4 \mathrm{~cm}$ apart from the testee to the reader of access control.In the experiment for positioning function, the position delay time of system is less than 9 seconds, and every position result is correct in all areas .All examination results showed that all the following functions as personnel tracking, access control, vital signs monitoring, abnormal radio alarming have been realized in the monitoring system.
\end{abstract}

Keywords-Wearable;Positioningcard;Access control ; Vital signs monitoring ; Radio alarming

\section{INTRODUCTION}

It 's required that specific monitoring system should be capable to positioning the controlled personnel within monitoring area, meantime the staff location and status information could be sent to the monitoring center $[1,2]$. The positioning signal card not only be used to transmit the controlled personnel status monitoring information, but also be easy to carry and anti- removed. To this end, we learn from the current concept of IT development in the field of wearable devices [3-6], developed a kind of wearable wireless anti-removed identification positioning card system. This paper describes experimental works and relevant trials research of the wearable wireless anti-removed identification positioning card system (WAIPCS). Experiment results showed that all the following functions as personnel tracking, access control, vital signs monitoring, abnormal radio alarming have been realized in the monitoring system.

\section{PRINCIPLE OF THE EXPERIMENT DEVICE}

In the system, AST9D01L is used to as the core of the heart rate sensor, and Texas instruments' chip MSP430 series with CC1100 together served as wireless communication device[7-8]. Other key components include of anti-removed alarm button, heart rate detection sensor, alarm status indicator, and RFID personnel identification cards is be integrated too. The principle diagram of the system is shown in Fig .1.

WAIPCS includes the following six sections:

(1) Heart rate sensor module: every heart beat pulse could cause the faint change in pulse pressure of blood capillary and lead to a skin blood flow. Use reflective photoelectric sensor extracting and processing the signals, the body's heart rate information could be gotten. In order to reduce the measurement time and improve test accuracy, the cycle of weak signal peak is first tested and the heart rate is obtained [9].

(2) Core Module: This module is used for the communication control system. On the one hand it is connected to the heart rate sensor via SPI communication interface which could get the heart 
rate value accurately, on the other hand by simulating the CC1100 SPI interface can also send or receive data to ensure the communication process.

(3) Wireless transmitter module: data sent from the core chip is received by wireless transmitter module is sent out. In work manner of Half-duplex (FSK), Wireless transmitter module could avoid sending the same frequency at the same time and work stably and reliably. Based on signal strength detection function of the transmitter module, the location of personnel in the area could be aid in determining[10].

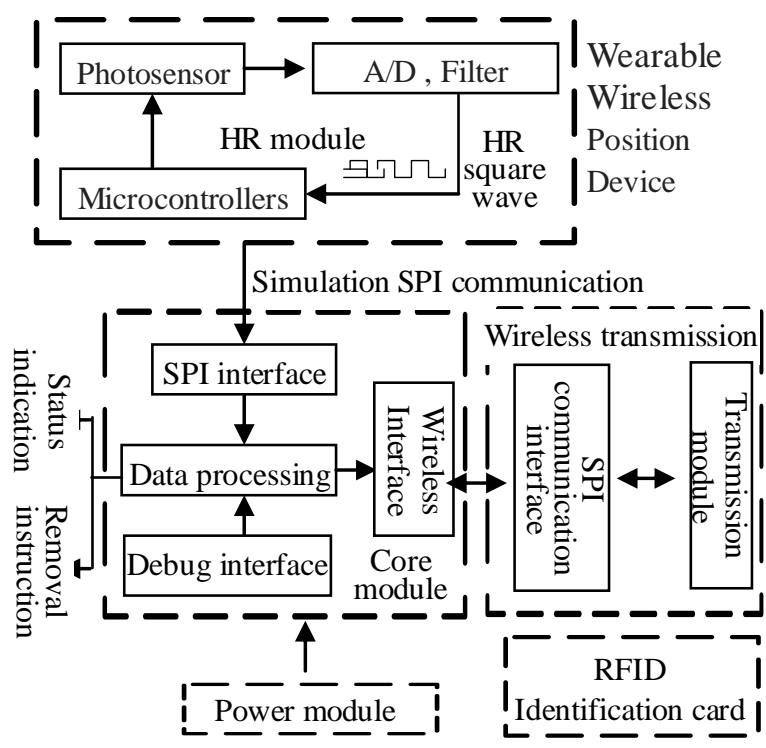

Figure 1. The principle diagram of WAIPCS

(4)Power supply module: based on the size of the wearable devices to select the battery capacity rationally which ensure to supplies voltages for WAIPCS, and ensure the battery be normal operation both in charging and in working.

(5) Antenna : used for transmitting and receiving radio waves, connecting base station with RF system to do positioning and communication .

( 6 ) Access Card: be Integrated into the wearable devices, two kind cards as positioning cards and access cards then becomes one.

In the software design of the system, the important module includes heart rate test module program and wireless data transceiver program. The former program flow chart is shown in Fig .2.

\section{EXPERIMENT CONTENT}

\section{A. Test of Heart rate reading function}

The Experiment device is prototype of WAIPCS shown in Fig .3 In Center is a photosensitive element, and both sides are the green light emitting diodes. The upper and lower slotted holes are designed for installation the strap. When be in contact with the skin or be put on the wrist, the diode LED will automatically give out light. Through contact with the skin doing the reflection and scattering of light, and the signal is received by the photosensitive element. Then it is sent to MCU after be amplified and be filtered, in last Heart rate data as the result is outputted through the SPI interface.

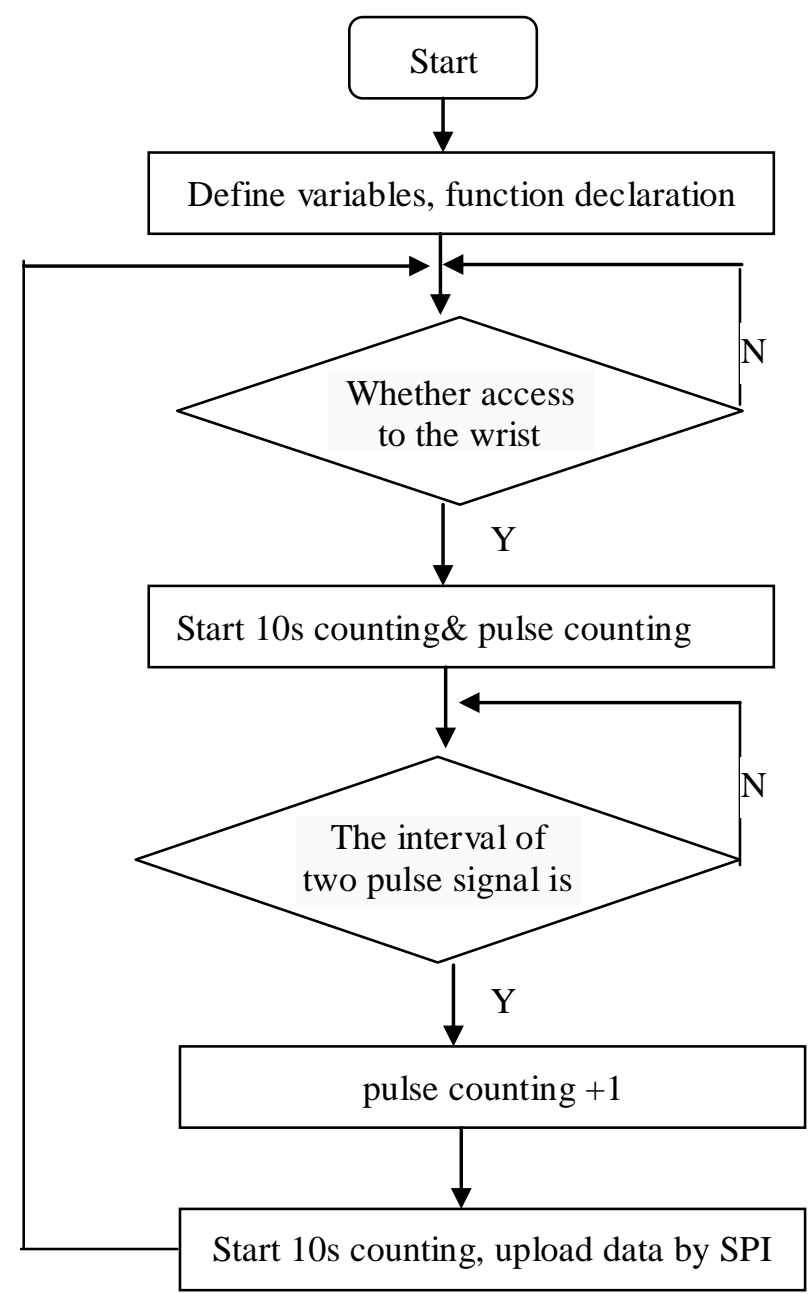

Figure 2. Flow chart of heart rate test module

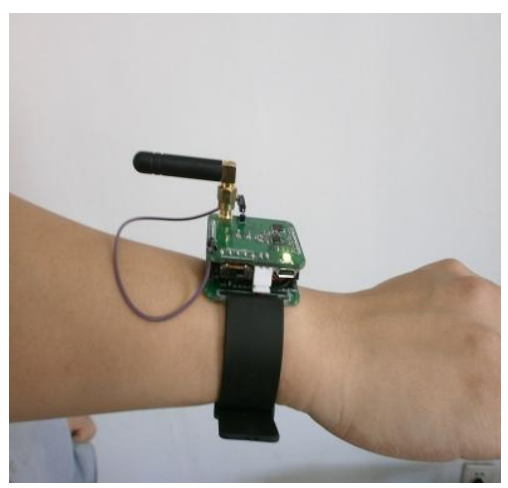

Figure 3. The prototype of WAIPCS

The main contents of the heart rate reading function test are as described below.

(1) To examine the system function of automatic monitoring and recognition its work environment. 
AS an prototype of WAIPCS in worn, its heart rate reading device should be in working condition and its power is on. Whereas its heart rate reading device should be turned off automatically

(2) The heart Rate waveforms could be watched by oscilloscope, heart rate reading. Normal heart rate in healthy adults is 60 to 110 times / min, so a correct test result should fall within the range.

\section{B. Test basic function of prototype of WAIPCS}

The main contents of tests are as described below.

(1) Instruction and state recognizing as the anti-removed identification positioning card in normal worn.

(2) Instruction and state recognizing at the occurrence of non-authorized events (such as illegal off locator card, etc.);

(3) To test the function of access control system in inlet and outlet as a testee passing with anti-removed identification positioning card.

\section{Experiment for positioning function}

Positioning function of the system based on comprehensive analysis and estimate fixed position known base station, which can make use of a small number of base stations to determine label area. Positioning principle is shown as Fig .4.

When a testee activities in the designated area, the system could do real-time tracking his activities based on WAIPCS instruction and reporting his place.
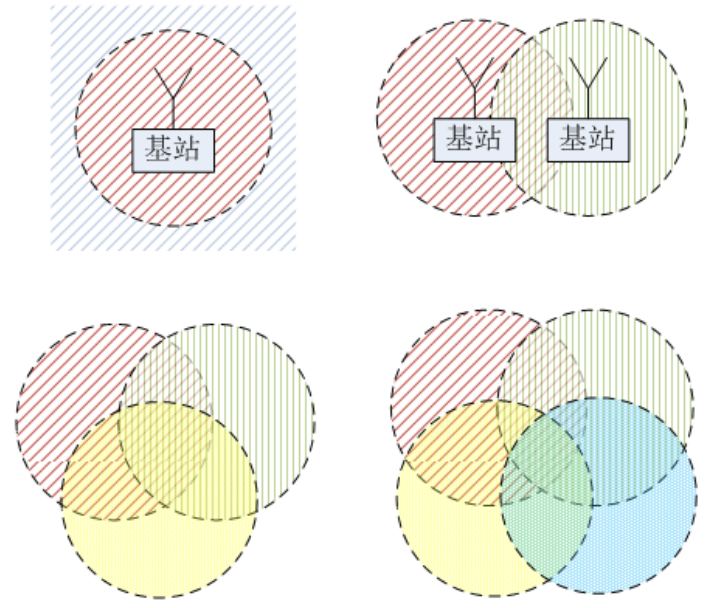

Figure 4. Positioning principle of WAIPCS

\section{EXPERIMENT RESULT AND}

\section{ANALYSIS}

\section{A. Experiment result of heart rate reading}

When the palm is placed at a distance of fewer $3 \mathrm{~cm}$ apart to heart rate sensor, the LED would give out light which is automatically adjusted the light intensity to reduce power consumption according to the distances. When the device is not in the state of being in worn, the lower plate light tube will automatically shut down. Fig .5 shows the experiment device of reading heart rate, which includes two states.

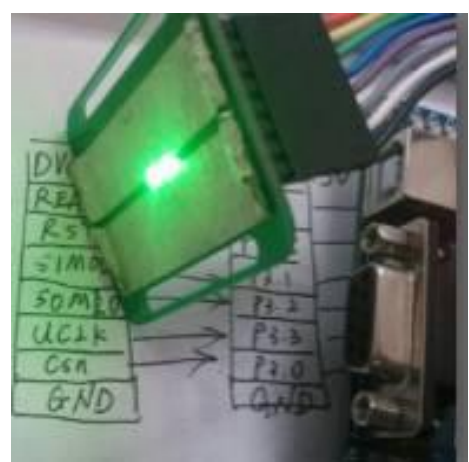

Figure 5-1. State of reading heart rate

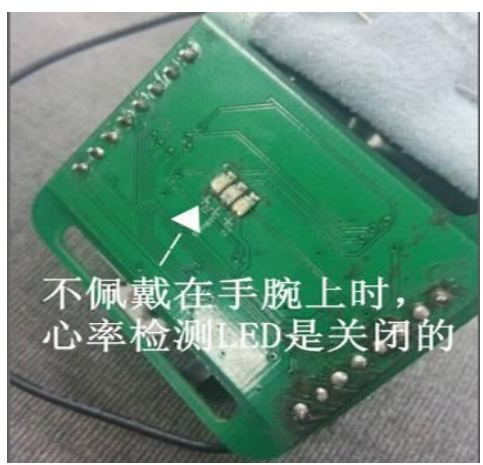

Figure 5-2. State of shutdown

Figure 5. Experiment device of reading heart rate

Fig .6 is a heart rate waveforms produced by WAIPCS output to oscilloscope. The upper curve is the unfiltered heart rate and the lower graph is heart rate square wave after filtered. Normal heart rate by experiments shows that adult is 60 to 80 times / min and females is a fewer faster whereas older man is a fewer slowly. Experimental results are consistent with the actual human heart rate and meet the design requirements.

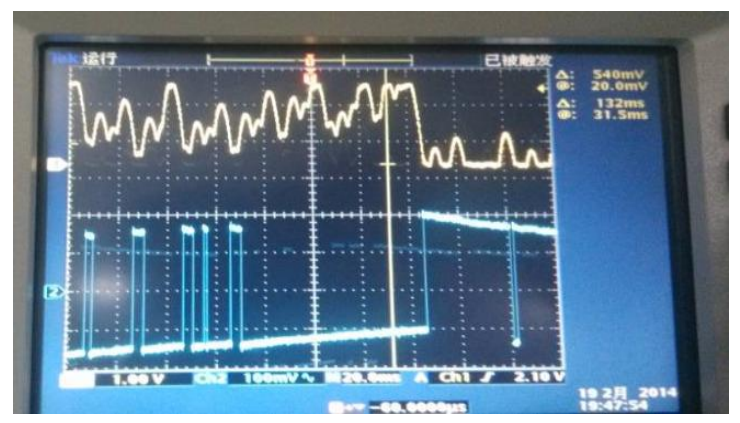

Figure 6. Experiment of heart rate waveforms

\section{B. Test basic positioning of WAIPCS}

If a testee tries to pull off the harness of WAIPCS 
wrapped around his wrist, the alarm message would be immediately sent to the monitoring center which alert system with a non- authorized event.

Fig .7 is a test scenarios of a testee passing access control system with WAIPCS in worn. Experiment shows that the system could read the Id information reliably within the distance $4 \mathrm{~cm}$ apart from the testee to the reader of access control .

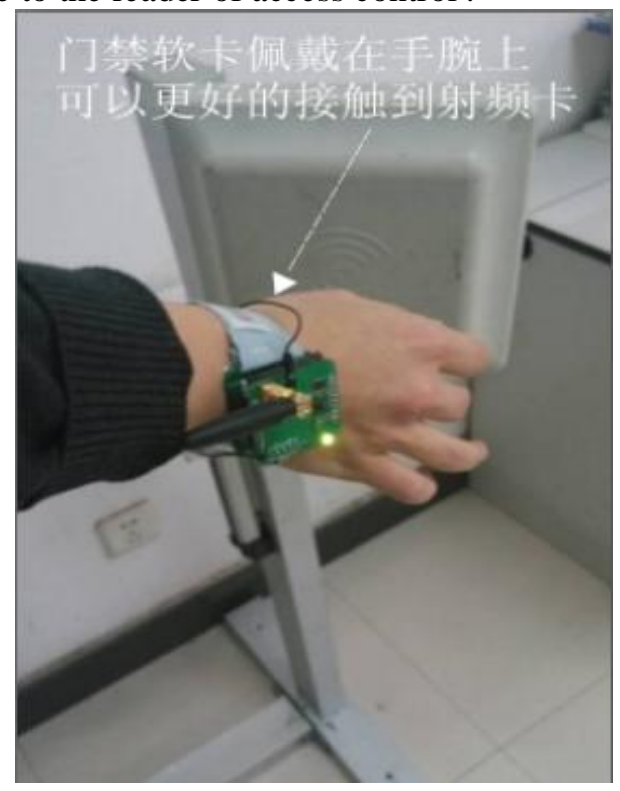

Figure 7. WAIPCS is being read by access control system

\section{Function tests of positioning system.}

Fig .7 is a schematic view of the testee motion based on tracking the testee and analyzing data, the testee's behavior identification results can be got. In first he appears in zone-1 and stays in the the positioning 69 seconds ; then appears in zone- 2 and stays 60 seconds; then appears in the zone-3 and stay 50 seconds; then appears in the zone- 4 and stay 42 seconds; at last the testee appears zone-5 and stay 64 seconds. The experiment shows the position delay time of system is less than 9 seconds, and every position result is correct in all areas .

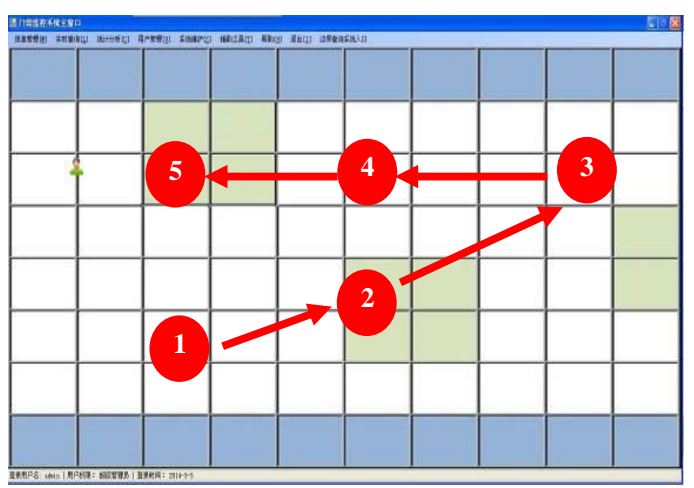

Figure 7. Sketch map of tracking a testee activities

\section{CONCLUSION}

WAIPCS integrated heart rate detection module, wireless transceiver modules, power modules, with a common RF card access control functions and personnel positioning. Experiment result shows the basic design requirements of WAIPCS has been satisfied Not only the function monitoring the personnel of abnormal behavior and monitoring abnormal state has been implemented, but also the requirement of $\mathrm{RF}$ card system miniaturization and being wearable have been realized. These achievements mark the study of our particular application monitoring tools has reached a new level of technology.

\section{REFERENCES}

[1] HE Shengping, Li Hongtao . Study of A Safe Approach to Wireless Image \& Video Transmission, 2012 (4) : 348 350

[2] HE Shengping, Wu Ruina, Analysis of underground vibration signals based on BSS, Technical Acoustics, 2011(4): 340 343

[3] YU Nanxiang, CHEN Dongyi, HIAHOU Shiji . wearable computing human computer interaction on-site task assistant wearable properties, 2012(4): 13-20

[4] Pentland, A. Looking at people: sensing for ubiquitous and wearable computing.IEEE Transactions on Pattern Analysis and Machine Intelligence, Jan. 2000, Vol.22(1), pp.107-119

[5] Zhang, Kuan ; Liang, Xiaohui ;etc. PHDA: A priority based health data aggregation with privacy preservation for cloud assisted WBANs .Information Sciences ISSN: 0020-0255 ; DOI: 10.1016/j.ins.2014.06.011

[6] Xybernaut Corp. Xybernaut Mobile/Wearble Computing Solutions Garner Attention of City, County Managers , Business Wire, Oct 19, 2004

[7] Yang, Bifeng ; Zhan, Yanjun ; Ma, Shangchang,Wireless module hardware design based on CC1100,2012 9th International Conference on Fuzzy Systems and Knowledge Discovery, May 2012, pp.2278-2281

[8] Zhang, Li-Qiang ; Han, Xin-Min.Research on design and optimization of municipal pipe networks wireless monitoring system. 2011 IEEE 18th International Conference on Industrial Engineering and Engineering Management, Sept. 2011, Vol.Part 1, pp.618-623

[9] AST9D01L Intelligent pulse monitoring IC , http://adsmart.com.cn/ ast9D01L.html

[10] Guo, Xiaolin ; Song, Zheng ; etc.An inter-device positioning method based on inertial sensors and wireless signal strength.2013 3rd International Conference on Consumer Electronics, Communications and Networks, Nov. 2013, pp.121-124 